\title{
TRPM1 Forms Complexes with Nyctalopin In Vivo and Accumulates in Postsynaptic Compartment of ON-Bipolar Neurons in mGluR6-Dependent Manner
}

\author{
Yan Cao, Ekaterina Posokhova, and Kirill A. Martemyanov \\ Department of Neuroscience, The Scripps Research Institute, Jupiter, Florida 33458
}

Synaptic transmission between light-sensory photoreceptor cells and downstream ON-bipolar neurons plays an important role for vertebrate vision. This process is mediated by the G-protein-coupled receptor pathway involving glutamate receptor mGluR6 and effector channel TRPM1. The signal transmission occurs on a rapid timescale; however, the molecular organization that ensures timely signaling in this cascade is unknown. Genetic studies in human patients and animal models reveal that ON-bipolar cell signaling depends on the synaptic protein nyctalopin. We have conducted a proteomic search for proteins associated with nyctalopin in the mouse retina and identified TRPM1 as the binding partner. We further demonstrate that nyctalopin additionally interacts with mGluR6 receptor. Disruption of mGluR6 prevented targeting of TRPM1 to the postsynaptic compartment of ON-bipolar neurons. These results reveal a unique macromolecular organization of the mGluR6 cascade, where principal signaling components are scaffolded by nyctalopin, creating an organization essential for the correct localization of the signaling ensemble and ultimately intact transmission of the signal at the first visual synapse.

\section{Introduction}

Synaptic transmission between neurons of the retina is critical for vertebrate vision. Absorption of light by photoreceptors hyperpolarizes the cells and leads to the suppression of neurotransmitter glutamate release from their axonal terminals. Across the synapse, changes in glutamate concentration are sensed by the second-order neurons, bipolar cells (BCs). Rod photoreceptor neurons are active under low-illumination conditions and signal predominantly to the $\mathrm{ON}$ type of bipolar cells (ON-BCs) that depolarize in response to light-evoked reduction in synaptic glutamate concentration (Sharpe and Stockman, 1999; Taylor and Smith, 2004). The ON-BCs play an essential role in dim vision, and their dysfunction is one of the leading causes of the congenital types of night blindness (Berger et al., 2010).

It has been established that glutamate reception of ON-BCs is mediated by the mGluR6 receptor (Nakajima et al., 1993; Masu et al., 1995) and involves the activation of a G-protein, Go (Nawy, 1999; Dhingra et al., 2000). Inactivation of either mGluR6 (Masu et al., 1995; Maddox et al., 2008) or Go (Dhingra et al., 2000) in mice leads to a complete absence of ON-BC responses to light. Genetic studies revealed that synaptic transmission to ON-BCs is

\footnotetext{
Received April 4, 2011; revised June 17, 2011; accepted June 22, 2011

Author contributions: Y.C. and K.A.M. designed research; Y.C. and E.N.P. performed research; Y.C., E.N.P., and K.A.M. analyzed data; K.A.M. wrote the paper.

This work was supported by NIH Grants EY018139 and DA026405. We recognize the Center for Mass Spectrometry and Proteomics at the University of Minnesota and various supporting agencies, including the National Science Foundation for Major Research Instrumentation Grants 9871237 and NSF-DBI-0215759, which were used to purchase the instruments described in this study. We thank Dr. Mathew D. Stone for the help with the protein identification experiments.

Correspondence should be addressed to Dr. Kirill A. Martemyanov, Department of Neuroscience, The Scripps Research Institute, 130 Scripps Way, 3C2, Jupiter, FL 33458. E-mail: kirill@scripps.edu.

DOI:10.1523/JNEUROSCI.1682-11.2011

Copyright $\odot 2011$ the authors $\quad 0270-6474 / 11 / 3111521-06 \$ 15.00 / 0$
}

also abolished in mice carrying inactivating mutation in the nyctalopin gene (Gregg et al., 2003). Nyctalopin is a membrane-anchored protein that contains several leucine-rich repeat domains, ubiquitous structural motifs that are important for protein-protein interaction (Poopalasundaram et al., 2005). In the retina, nyctalopin is expressed at the dendritic tips of ON-BCs, where it closely colocalizes with mGluR6, forming prominent synaptic puncta (Morgans et al., 2006; Gregg et al., 2007). Single-cell electrophysiological studies demonstrated that $\mathrm{ON}-\mathrm{BC}$ lacking nyctalopin are insensitive to direct application of glutamate, suggesting the involvement of this protein in modulating the components of the signal transduction pathway (Gregg et al., 2007).

One of the most significant recent breakthroughs in understanding $\mathrm{ON}-\mathrm{BC}$ signaling has been the identification of the TRPM1 as the main effector channel that mediates ON-BC responses to mGluR6 activation (Morgans et al., 2009; Shen et al., 2009; Koike et al., 2010). Like mGluR6 and nyctalopin, TRPM1 is specifically enriched at dendritic tips, and its elimination in mice completely abolishes ON-BC responses. Genetic association studies indicate that mutations in TRPM1 (Audo et al., 2009; van Genderen et al., 2009; Nakamura et al., 2010), mGluR6 (Zeitz et al., 2005; O'Connor et al., 2006), and nyctalopin (Bech-Hansen et al., 2000; Leroy et al., 2009) account for the majority of congenital night blindness cases in human patients. However, beyond general knowledge regarding the identity of the main players involved in $\mathrm{ON}-\mathrm{BC}$ responses to light, our understanding of the mechanistic details pertaining to the organization of the signaling pathway is very limited.

In this study, we report identification of TRPM1 and mGluR6 as nyctalopin binding partners. We further establish that the postsynaptic accumulation of TRPM1 complex is dependent on mGluR6. These studies indicate that the three main components of the $\mathrm{ON}-\mathrm{BC}$ signaling pathway are linked together in a single macromo- 

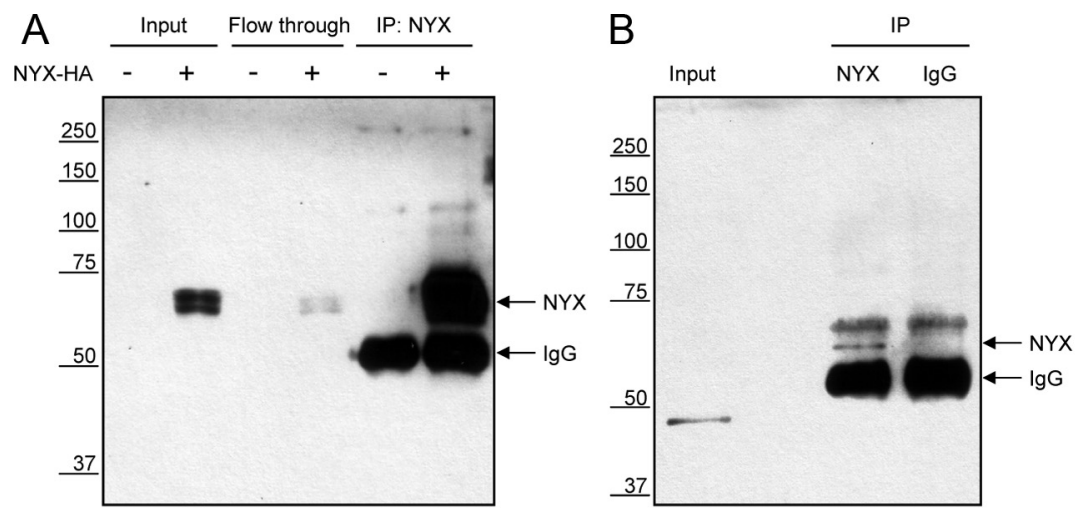

C sp|p83503|NYX_MOUSE, 52,431.9 Da Nyctalopin Mus musculus

6 unique peptides, 6 unique spectra, 6 total spectra, 101/476 amino acids (21\% coverage)

MLILLLHAVV FSLPYTRATE ACLRACPAAC TCSHVERGCS VRCDRAGLQR VPQEFPCEAA SIDLDRNGLR ILGERAFGTL PSLRRLSLRH NNLSEITPGA EKGLPRLAEL RLAHNGELRY LHVRTFAALG RLRRLDLAAC RLESVPERLL AELPALRELT AFDNLFRRVP GALRGLANLT HAHFERSRIE AVASGSLLGM RRLRSLSLQA NRVRAVHAGA FGDCGALEDL LINDNLLATL PAAAFRGLRR. LRTLNLGGNA LGSVARAWFS DLAELELLYL DRNSITFVEE GAFQNLSGLI ALHLNGNRLT VLSWAAFQPG FFLGRLFLFR NPWRCDCQLE WLRDWMEGSG RVADVACASE GSVAGQDLSQ VVFERSSDGL CVDPDELNFT TSSPGPSPEP VATTVSRFSS LLSKLLAPRA PVEEVANTTW ELVNVSLNDS FRSHAVMVFC YKATFLFTSC VLLSLAQYVV VGLQRE

\begin{tabular}{|c|c|c|c|c|c|c|}
\hline Peptide sequence & $\begin{array}{c}\text { Precursor } \\
\mathrm{m} / 2\end{array}$ & Charge & $\triangle P P M$ & $\begin{array}{l}\text { Peptide } \\
\text { identification } \\
\text { probability }\end{array}$ & $\begin{array}{l}\text { SEQUEST } \\
\text { XCorr score }\end{array}$ & $\begin{array}{l}\text { SEQUEST } \\
\text { DCn score }\end{array}$ \\
\hline AFGTLPSLR & 481.28 & 2 & -1.4 & $95.00 \%$ & 2.25 & 0.14 \\
\hline AVHAGAFGDCGALEDLLLNDNLLATLPAAAFR & $1,099.56$ & 3 & 1.3 & $95.00 \%$ & 4.47 & 0.522 \\
\hline IEAVASGSLLGMR & 660.35 & 2 & 0.56 & $95.00 \%$ & 2.61 & 0.365 \\
\hline LTVLSWAAFQPGFFLGR & 955.52 & 2 & 0.5 & $95.00 \%$ & 3.21 & 0.426 \\
\hline TLNLGGNALGSVAR & 671.88 & 2 & 0.63 & $95.00 \%$ & 2.35 & 0.325 \\
\hline VPQEFPCEAASIDLDR & 923.93 & 2 & -2.6 & $95.00 \%$ & 1.78 & 0.308 \\
\hline
\end{tabular}
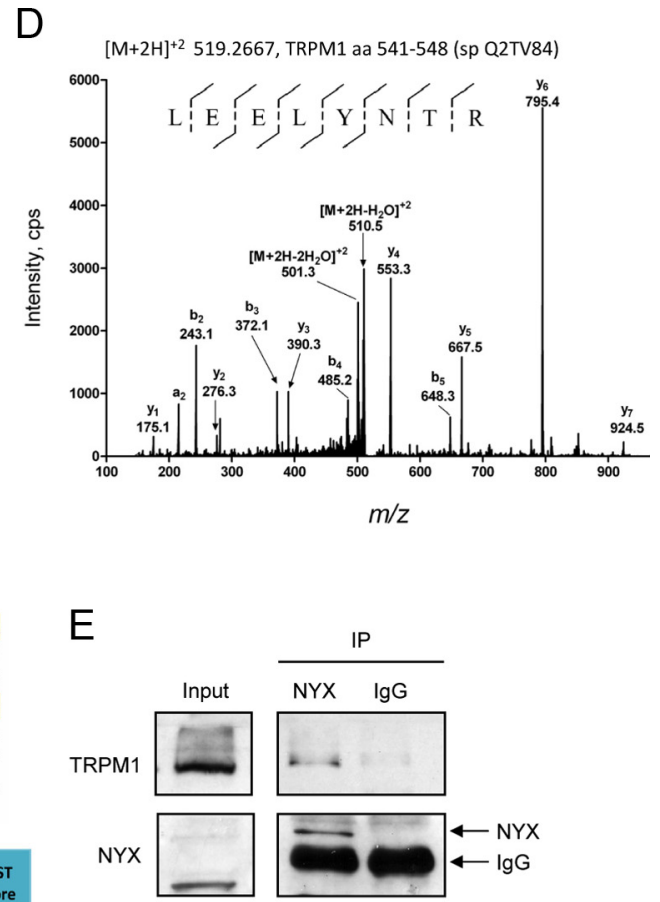

Figure 1. Identification of nyctalopin binding partners in the retina. $\boldsymbol{A}$, Characterization of anti-nyctalopin antibodies. Cells were transfected with a construct encoding the full-length mouse nyctalopin gene (NYX-HA) and used to prepare lysates. Upon lysis in the IP buffer, nyctalopin was immunoprecipitated with anti-nyctalopin antibodies conjugated to protein $\mathrm{G}$ beads. Proteins in the total lysate (Input), in fraction unbound to the beads (Flow-through), and upon elution from the antibody-conjugated beads (IP: NYX) were resolved by the SDS-PAGE and detected by Western blotting with anti-nyctalopin antibodies. $\boldsymbol{B}$, Detection of nyctalopin in total retina lysates (Input) and after affinity purification on beads conjugated to anti-nyctalopin antibodies (IP). Nonimmune sheep lgG was used as a control for nonspecific binding. The positions of nyctalopin (NYX) and lgG are indicated by the arrows. Note that nonspecific band of lower molecular weight recognized by the antibodies in retinal lysates is not immunoprecipitated. $C$, List of peptides corresponding to nyctalopin sequence found in the eluate fraction from the immunoprecipitation reaction conducted using anti-nyctalopin antibodies and mouse retinas. Mapping of the peptides in the nyctalopin sequence is shown in yellow. Peptides were identified by MS/MS sequencing on an LTQ-Orbitrap mass spectrometer, and their identification probability was analyzed by Sequest software. Minimum $95 \%$ confidence interval was used to assign significant hits. $\boldsymbol{D}$, MS/MS fragmentation spectrum of a TRPM1 peptide. Precursor peptide was subjected to collision induced dissociation fragmentation, and the resulting ions were used to deduce the amino acid sequence shown above. $\boldsymbol{E}$, TRPM1 coimmunoprecipitates with nyctalopin from mouse retinas. Mouse retinas were lysed and used to immunoprecipitate nyctalopin. Coprecipitation of TRPM1 was detected by Western blotting.

lecular complex, providing an insight into the compartmentalization of the postsynaptic signaling machinery of retina ON-BC neurons.

\section{Materials and Methods}

Antibodies, DNA constructs, and mouse strains. Antibodies against mouse recombinant nyctalopin (amino acids 19-450), TRPM1 (amino acids 1423-1622), and mGluR6 (amino acids 853-871) were generated in sheep. Antibodies were affinity purified on the antigen-containing column and used throughout the study. The specificity of all antibodies has been verified by their ability to recognize cognate proteins transiently expressed in transfected HEK293T cells and to precipitate them from native retinas as determined by mass spectrometry. For double-immunolabeling experiments, guinea pig anti-mGluR6 antibodies were used (gift from Dr. Furukawa, Osaka Bioscience Institute, Osaka, Japan). The following commercial antibodies were used: mouse anti- $\beta$-actin (Sigma); mouse anti-CtBP2 (BD Biosciences); mouse anti-HA (Millipore); and mouse anti-c-myc (Roche).

Cloning of rat mGluR6 was described previously (Cao et al., 2009). The C-terminal HA-tagged mouse nyctalopin was amplified from a mouse cDNA clone (clone ID 5362608, Open Biosystems) and cloned into a pcDNA3.1/V5-His-TOPO (Invitrogen) vector. C-terminal HA or tandem affinity purification (TAP) (Bürckstümmer et al., 2006) tags were appended before the stop codon by PCR. To obtain a N-terminal, c-myc-tagged, fulllength mouse TRPM1 expression construct, TRPM1(349-4869) was amplified from mouse cDNA clone (clone ID 100068120, Open Biosystems), supplemented with TRPM1(1-348) amplified from mouse TRPM1 cDNA clone (clone ID A930013B11, imaGenes) and cloned into a pcDNA3.1/V5His-TOPO (Invitrogen) vector.

nob3 and nob4 mouse strains were obtained from The Jackson Laboratory and the NIH Neurogenomics Project, respectively. Both male and female mice (1-3 months old) were used in the study. Mice were maintained on a diurnal $12 \mathrm{~h}$ light/dark cycle. Procedures involving mice strictly followed NIH guidelines and were approved by the Institutional Animal Care and Use Committee at the University of Minnesota.

Preparative immunoprecipitation and mass spectrometry. Whole retinas were removed from mice, lysed by sonication in $1 \%$ Triton X-100containing buffer, and subjected to immunoprecipitation as previously described (Martemyanov et al., 2005; Cao et al., 2009). The beads were washed three times with ice-cold immunoprecipitation (IP) buffer, and proteins were eluted with $50 \mu$ l of SDS sample buffer (62 mu Tris, $10 \%$ glycerol, $2 \%$ SDS, and 5\% $\beta$-mercaptoethanol). Resulting samples were allowed to enter the SDS-PAGE gel by applying $\sim 150 \mathrm{mV}$ for $15-20 \mathrm{~min}$. Gels were fixed with using $5 \%$ acetic acid in $50 \%$ methanol, stained by NOVEX colloidal blue (Invitrogen). Stained areas were cut out, digested with trypsin (Promega), and alkylated as described previously (Shevchenko et al., 2006). The resulting peptide mixtures were desalted, resolved by highpressure liquid chromatography, and analyzed using LTQ-Orbitrap XL mass spectrometer, as previously described (Posokhova et al., 2011).

Cell culture, immunoprecipitation, pull down, and Western blotting. HEK293T cells were cultured at $37^{\circ} \mathrm{C}$ and $5 \% \mathrm{CO}_{2}$ in DMEM supplemented with $10 \% \mathrm{FBS}$, and $4 \mathrm{~mm}$ L-glutamine. Cells were transfected at 

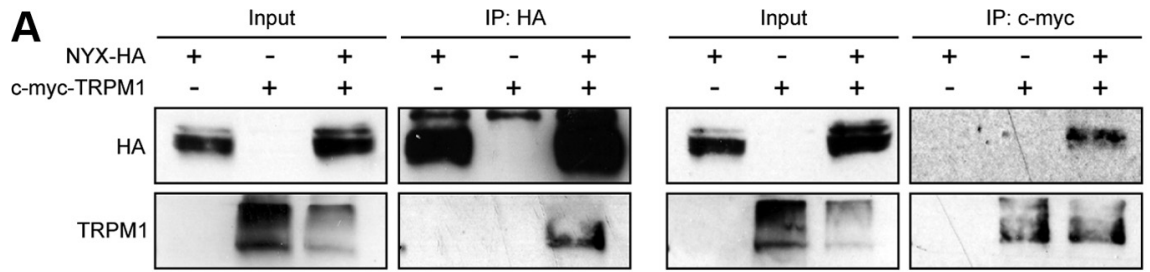

B
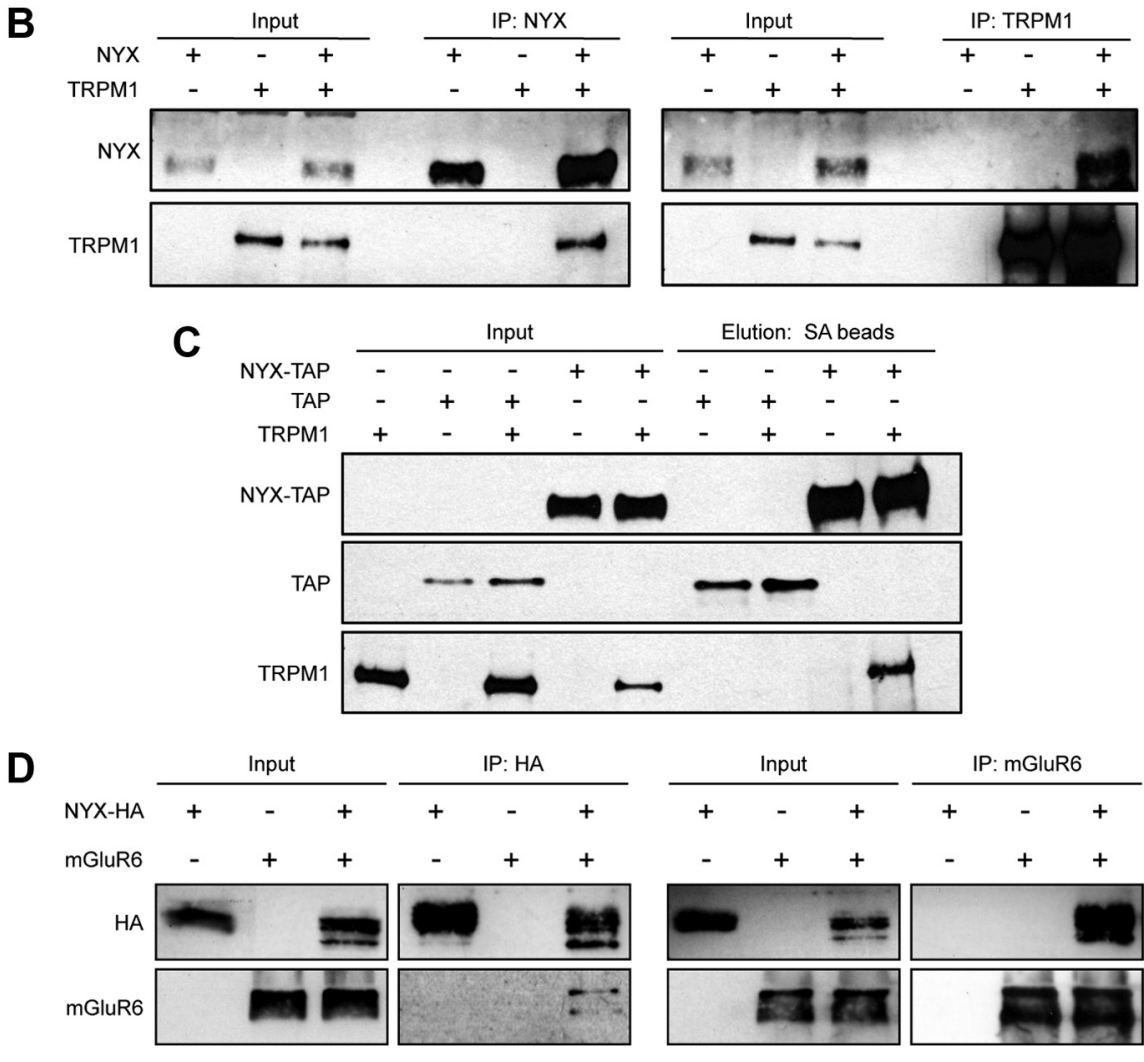

D

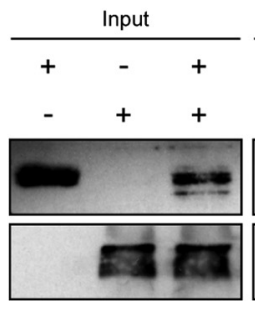

Figure 2. Nyctalopin binds to mGluR6and TRPM1 in cotransfected cells.A,TRPM1-nyctalopin interaction. Cultured HEK293T cells were transfected with constructs encoding HA-tagged nyctalopin (NYX-HA) or c-myc tagged TRPM1 (c-myc-TRPM1) alone or in combination, as indicated. Proteins present in total lysates (Input) were detected by Western blotting. Cellular lysates were subjected to immunoprecipitation with either anti-HA (left) of anti c-myc (right) antibodies, and proteins retained on the beads (IP) were detected by Western blotting with indicated antibodies. B, Coimmunoprecipitation of untagged nyctalopin with TRPM1 from HEK293T cells with specific antibodies. $\boldsymbol{C}$, Nyctalopin fused with TAP module pulls down TRPM1 from HEK293T lysates using streptavidin agarose (SA) beads. D, mGluR6 - nyctalopin interaction. The experiment was conducted exactly as described in $A$, except cells were transfected with mGluR6 instead of TRPM1 and reverse immunoprecipitation reaction was conducted with anti-mGluR6 antibodies.

$\sim 70 \%$ confluency using Lipofectamine LTX (Invitrogen) and harvested $24 \mathrm{~h}$ later. Cells were lysed in ice-cold PBS IP buffer by sonication, and lysates were cleared by centrifugation at 14,000 rpm for $15 \mathrm{~min}$. For immunoprecipitation experiments, the supernatant was incubated with $10 \mu \mathrm{l}$ of protein G beads (GE Healthcare) and $5 \mu \mathrm{g}$ of antibodies on a rocker at $4^{\circ} \mathrm{C}$ for $1 \mathrm{~h}$. After three washes with PBS IP buffer, proteins were eluted with $50 \mu$ l of SDS sample buffer and analyzed by SDS-PAGE, followed by Western blotting using HRP-conjugated secondary antibodies and an ECL West Pico (Thermo Scientific) detection system. Pulldown experiments with TAP-tagged constructs were performed using Streptavidin Sepharose (GE Healthcare). Procedures replicated ones used for the IP experiments, except that Triton X-100 in cell lysis buffer was substituted for $0.5 \% \mathrm{E} 10 \mathrm{C} 12$ and proteins were eluted from the beads with $25 \mu \mathrm{l}$ of $3 \mathrm{~mm}$ biotin. For quantitative analysis, Western blotting was performed with IRDye 680- and IRDye 800-labeled secondary antibodies (Li-Cor Biosciences), and the signal was detected and quantified by an Odyssey Infrared Imaging System (Li-Cor Biosciences). The integrated intensity of each band of interest was measured in a corresponding channel with a top-bottom background setting. Integrated intensity of $\beta$-actin was used for data normalization.

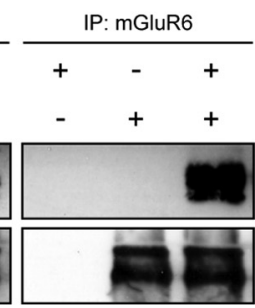

Immunohistochemistry. Immunohistochemical detection of proteins in retinal sections was performed as described previously (Cao et al., 2008). Dissected eyecups were fixed for $15 \mathrm{~min}$ with $4 \%$ paraformaldehyde, blocked with $10 \%$ donkey serum for $1 \mathrm{~h}$, and incubated with primary antibody in for $1 \mathrm{~h}$. After four washes, sections were incubated with fluorophoreconjugated secondary antibodies for $1 \mathrm{~h}$ before mounting in Fluoromount (Sigma). Images were taken by the Olympus Fluoview 1000 confocal microscope.

\section{Results}

\section{Identification of TRPM1 as nyctalopin} binding partner in the retina

To gain an insight into the functional role of nyctalopin at the molecular level, we have conducted a proteomic search for its binding partners in the retina. We began by developing antibodies to nyctalopin that would recognize native protein. Antibodies directed against the globular portion of the nyctalopin efficiently and specifically recognized nyctalopin expressed transiently in transfected HEK293T cells (Fig. 1A). Furthermore, when used for immunoprecipitation, these antibodies could substantially deplete nyctalopin from the cellular lysates and dramatically enrich the protein in the eluate fractions, demonstrating their utility for affinity purification of nyctalopin (Fig. 1A).

When tested in native retinas, nyctalopin antibodies failed to recognize specific bands in the total retina lysate. However, when used for immunoprecipitation the antibodies were able to pull down a 52 $\mathrm{kDa}$ protein that corresponds to the predicted molecular weight of nyctalopin and matches the migration position of nyctalopin expressed in HEK293T cells (Fig. 1B). This immunoprecipitation was specific as nonimmune IgG failed to pull down the band. We have next conducted a preparative immunoprecipitation reaction with 14 mouse retinas and subjected eluates to tandem mass spectrometric analysis. Tandem mass spectrometry (MS/ MS) sequencing of precursor peptides identified six unique peptides with at least 95\% confidence that precisely matched the sequence of Mus musculus nyctalopin derived from the database (Fig. 1C). Importantly, none of the nyctalopin peptides were detected in the control immunoprecipitation reaction where nonimmune IgG beads were used instead. These results allow us to conclude with confidence that our antibodies can specifically immunoprecipitate nyctalopin from native mouse retinas.

Examination of proteins pulled down with anti-nyctalopin antibodies identified the presence of TRPM1. A sequence of single, unique peptide matching to amino acids $541-548$ of mouse TRPM1 protein was deduced with high confidence by MS/MS fragmentation (Fig. 1D). Western blot analysis of samples from immunoprecipitation confirmed the presence of full-length TRPM1 protein in eluates where anti-nyctalopin antibodies were used for the pull-down (Fig. $1 E)$. Importantly, no TRPM1 protein was detected by either mass 
A
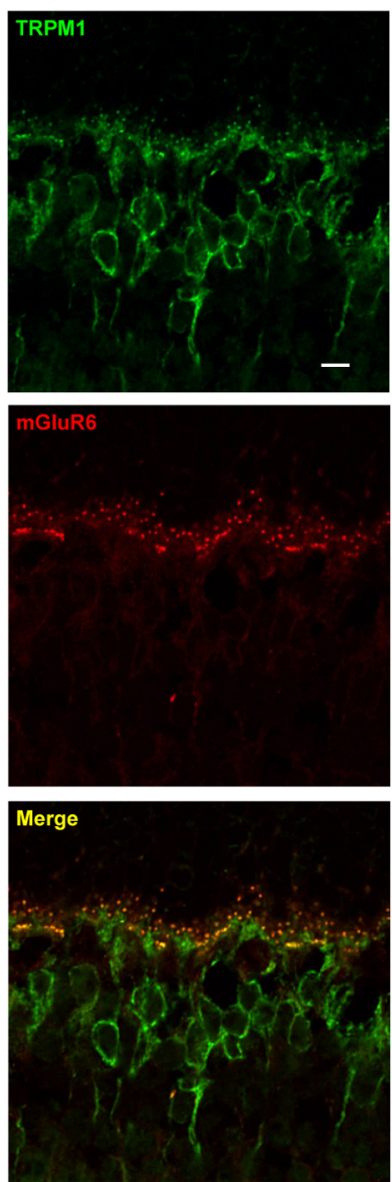

B
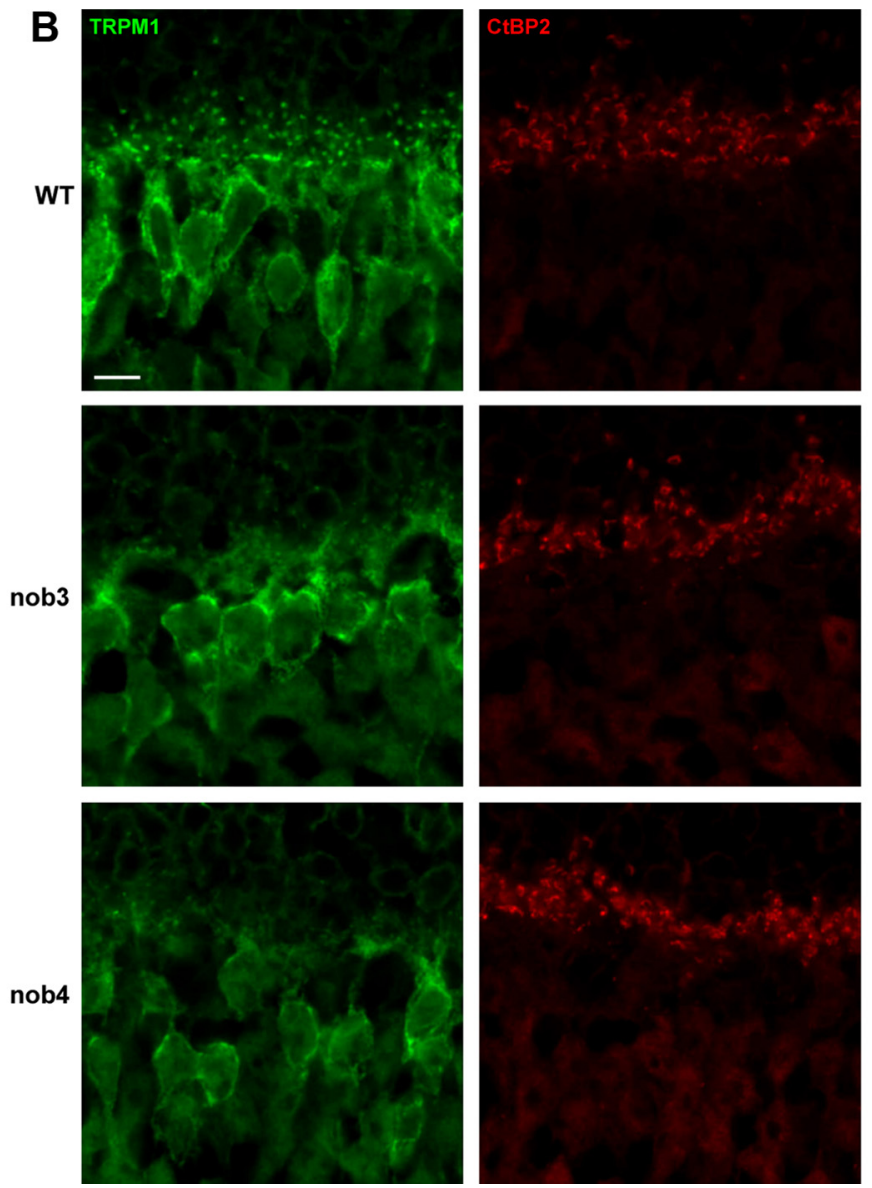
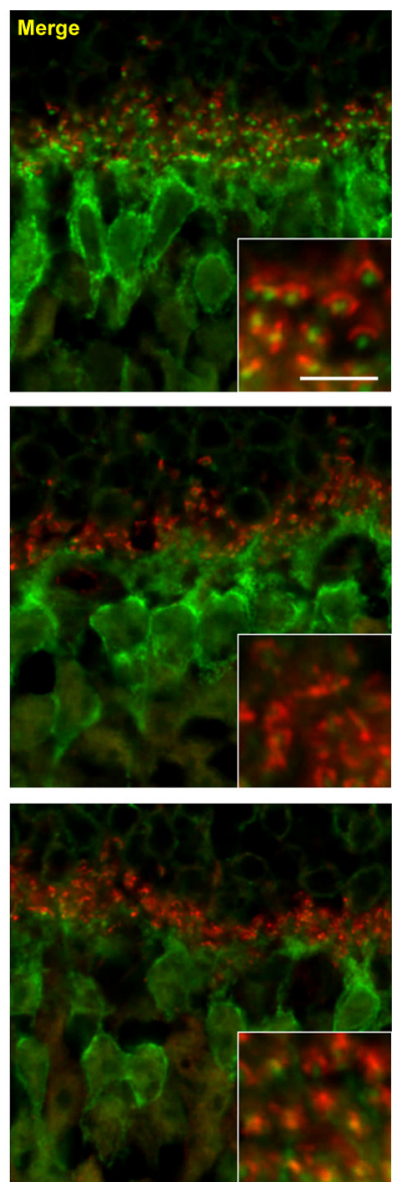

Figure 3. Targeting of TRPM1 to dendritic tips of ON-BCs requires mGluR6. $A$, Colocalization of TRPM1 and mGluR6 at dendritic tips of ON-BCs. Cross sections of mouse retinas were double stained with TRPM1 (green) and mGluR6 (red) antibodies, as described in Materials and Methods. Scale bar, $5 \mu \mathrm{m}$. B, TRPM1 is absent at the tips of the 0N-BC dendrites in retinas lacking mGluR6. Immunohistochemical analysis of TRPM1 localization in the outer plexiform layer of wild-type (WT), nob3, and nob4 retinas. Sections were double stained with TRPM1 (green) and presynaptic marker CtBP2 (red) antibodies. Insets show the outer plexiform layer at high magnification. Scale bar, $5 \mu \mathrm{m}$.

spectrometry or Western blotting in control experiments where nonimmune IgGs were used instead. These data indicate that nyctalopin specifically interacts with TRPM1 in native retinas.

\section{Nyctalopin forms complexes with both TRPM1 and mGluR6}

We next examined whether binding between nyctalopin and TRPM1 could be reconstituted in a heterologous expression system. First, epitope-tagged constructs encoding c-myc-TRPM1 and HAnyctalopin were cotransfected into mammalian HEK293T cells. We were able to effectively precipitate nyctalopin from the cellular lysates using anti-HA antibodies (Fig. 2A). Examination of the eluate fraction revealed the robust presence of c-myc-TRPM1 only in samples where nyctalopin was present, indicating specific coprecipitation of TRPM1. Conversely, in reciprocal immunoprecipitation experiment, nyctalopin was specifically present in the eluates when TRPM1 was pulled down with anti c-myc antibodies (Fig. 2 A). Similar results were obtained when specific antibodies were used to precipitate untagged proteins in both forward and reverse directions (Fig. $2 B$ ). Furthermore, when attached to the TAP protein module, nyctalopin, but not TAP alone, was able to effectively pull down TRPM1 from cellular lysates (Fig. 2C).

mGluR6 is the principle receptor of the ON-BC that controls closing of TRPM1 channel through the activation of the intracellular signaling cascade. Therefore, we next examined the possibility that nyctalopin could be additionally involved in the interactions with mGluR6. As is evident from Figure $2 D$, in cells cotransfected with both nyctalopin and mGluR6, but not in the cells transfected with only one protein, mGluR6 was effectively coprecipitated with nyctalopin in both forward and reverse immunoprecipitation reactions (Fig. 2D). These data indicate that nyctalopin can form protein-protein interactions with two principle components of the ON-BC signal transduction pathway: the receptor mGluR6 and the effector channel TRPM1.

\section{mGluR6 controls localization and expression of TRPM1}

Binding of nyctalopin to both mGluR6 and TRPM1 suggests that these proteins exist in a ternary complex. Because mGluR6 has been previously shown to target proteins associated [e.g., R7 regulators of G-protein signaling (RGS) proteins] (Cao et al., 2009) with it to the dendritic tips of ON-BCs, we have tested a possibility that mGluR6 also drives postsynaptic accumulation of TRPM1 channels. Therefore, we have next performed immunohistological studies to examine the role of mGluR6 in subcellular localization of TRPM1. Consistent with recent reports (Morgans et al., 2009; Koike et al., 2010), we found that TRPM1 staining was located on the plasma membrane and was specifically enriched at the tips of the ON-BC dendrites forming a characteristic punctate staining pattern, completely overlapping with mGluR6 immunofluorescence (Fig 3A). The TRPM1-positive puncta immediately opposed presynaptic photoreceptor terminals revealed by labeling with the synaptic ribbon marker CtBP2 (Fig. 3B). Strikingly, we found complete lack of 

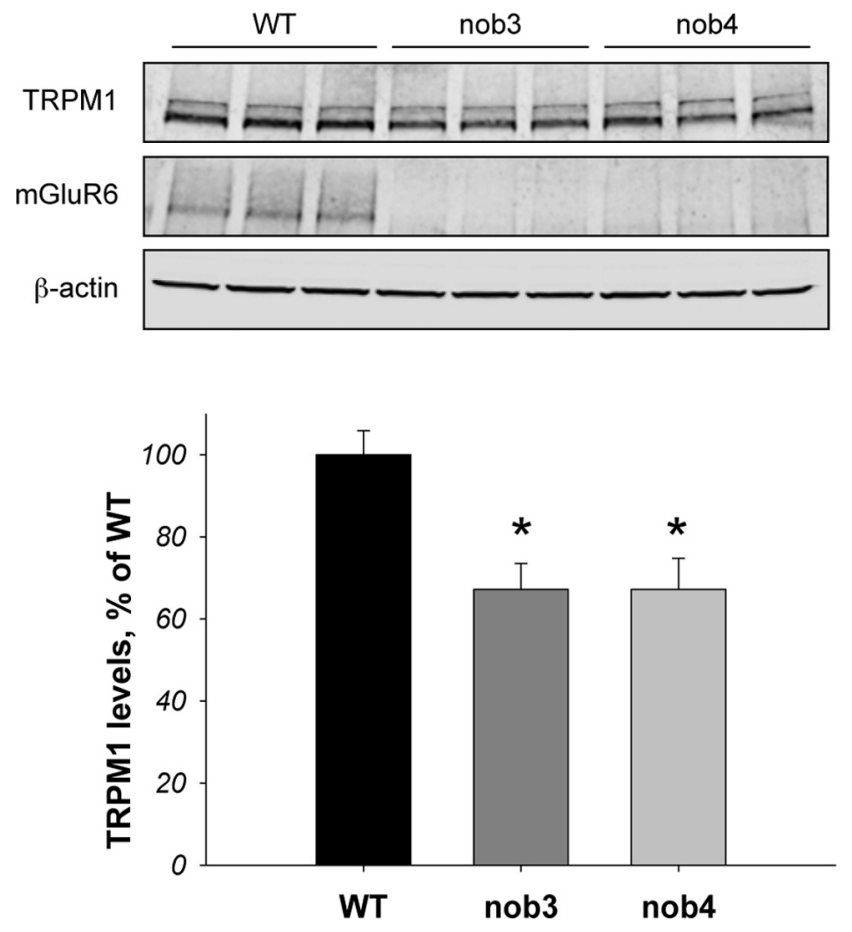

Figure 4. Regulation of TRPM1 expression level by mGluR6. Western blot analysis of protein expression in wild-type (WT), nob3, and nob4 retinas. Total retina lysates $(8.5 \mu \mathrm{g})$ obtained from three to four separate mice were loaded in each lane. Blots were probed with indicated antibodies, as described in Materials and Methods. $\beta$-actin detection was used as a loading control. Bottom, Quantification of the Western blotting data shown in the top panel. TRPM1 band intensities were determined by the 0 dyssey Infrared Imaging System and were normalized to $\beta$-actin content in the same sample. Values were reflected and plotted as the percentage of the level in WT retinas. Error bars represent SEM values. The asterisk indicates a statistically significant difference $(p<0.05)$, as revealed by Student's $t$ test. The experiment shown is representative of four independent experiments yielding similar results.

this punctate postsynaptic accumulation of TRPM1 in retinas of nob3 and nob4 mice that do not express mGluR6 protein (Fig. 3B). At the same time, the plasma membrane localization of TRPM1 in the mutant retinas was unchanged and largely resembled that observed in the wild-type samples. We have further noticed that mutant retinas consistently yielded lower fluorescence intensities when stained for TRPM1. This observation was confirmed by Western blotting that revealed a $30 \%$ reduction in the levels of TRPM1 protein in nob3 and nob4 mutant retinas lacking mGluR6 (Fig. 4). These observations indicate that macromolecular complex formation with mGluR6 is essential for the synaptic targeting and expression of TRPM1 in ON-BCs.

\section{Discussion}

The sign-inverting synapse between photoreceptors and ON-BC neurons provides a conversion of the light-induced hyperpolarizing response of photoreceptors into the excitatory depolarization of the downstream ON-BCs. The main players, as well as the key steps of this process at the postsynaptic site have now been established (Vardi et al., 2002; Okawa and Sampath, 2007; Morgans et al., 2010). In the hypothetical sequence of events, decrease in the glutamate release by the photoreceptors during light response leads to a reduction in mGluR6 activation, subsequent Go deactivation, and opening of the TRPM1 channels, which are kept closed in the dark by tonic mGluR6-G $\alpha$ o signaling (Vardi et al., 2002; Snellman et al., 2008). This makes TRPM1 the central effector channel that is ultimately responsible for providing ON-BC responses to light (Morgans et al., 2010).
Currently, the mechanisms governing gating and cellular regulation of TRPM1 in ON-BC neurons are completely unknown. What is known, however, is the remarkable speed with which ON-BCs mount the response. The TRPM1 channels fully open $<100 \mathrm{~ms}$ from the moment mGluR6 receptors detect changes in glutamate concentration (Field and Rieke, 2002). In comparison, the corresponding step in G-protein cascade of rod photoreceptors, deactivation of rhodopsin-transducin signaling and opening of the cGMP-gated channels, takes approximately five times longer (Pugh, 2006; Fain et al., 2010). These considerations suggest that postsynaptic G-protein cascade of ON-BCs is organized in a way that allows the streamlining of signal transmission from the mGluR6 to the TRPM1 channel. Indeed, mGluR6 cascade displays a remarkable compartmentalization with all key components being enriched at the tips of the ON-BC dendrites in immediate apposition to the photoreceptor axonal terminals.

One of the main results of our study is a demonstration that mGluR6 is required for targeting of TRPM1 channel to the dendritic tips, where it functions. In the absence of mGluR6, transmembrane channel TRPM1 is still able to reach plasma membrane but completely loses its accumulation at postsynaptic sites. This observation parallels our earlier report showing that targeting of RGS proteins to the dendritic tips is also mediated by the association of the RGS proteins with mGluR6. Together, these findings indicate the important role that mGluR6 receptor plays in subcellular targeting of pathway components in addition to its role in signaling.

The second key finding of this study is the demonstration of the macromolecular complex formation between principle components of the ON-BC signaling cascade: TRPM1 and mGluR6, which both physically associate with synaptic protein nyctalopin. We propose that assembly of this complex plays an essential role in enabling fast transmission of signal from mGluR6 to TRPM1, ensuring millisecond timing kinetics of $\mathrm{ON}-\mathrm{BC}$ responses to light. Because neither mGluR6 (Beqollari et al., 2009; Masuho et al., 2010) nor TRPM1 (Oancea et al., 2009; Koike et al., 2010; Lambert et al., 2011) appear to require nyctalopin for their functional activity in cells per se, we envision that the role of nyctalopin is to coordinate the assembly of the mGluR6-TRPM1 complex in the optimal configuration for signaling, which ultimately makes the fast signal transmission possible. Loss of nyctalopin in this scheme would disrupt the coupling of mGluR6 receptor to TRPM1, resulting in an inefficient response that would not reach the threshold level necessary for the generation of the light response. This situation is reminiscent of the invertebrate phototransduction cascade where the coupling of the G-proteincoupled receptor to the TRP effector channel requires participation of an auxiliary scaffolding protein [INAD (inactivation no afterpotential D)] that brings the components in the complex together and plays a critical role in visual signaling (Tsunoda and Zuker, 1999; Wang and Montell, 2007). Interestingly, several G-protein-coupled receptors carry extracellular nyctalopin-like leucine-rich repeat (LRR) domains (Hsu et al., 2000), and there is also a precedent for the involvement of LRR-containing proteins in allosteric modulation of the ion channel properties (Yan and Aldrich, 2010). These observations suggest a possible general mechanism of synaptic signaling where numerous LRR proteins (Ko and Kim, 2007) could be involved in modulating signal transmission events from G-proteincoupled receptors to TRP channels.

\section{References}

Audo I, Kohl S, Leroy BP, Munier FL, Guillonneau X, Mohand-Saïd S, Bujakowska K, Nandrot EF, Lorenz B, Preising M, Kellner U, Renner AB, Bernd A, Antonio A, Moskova-Doumanova V, Lancelot ME, Poloschek CM, Drumare I, Defoort-Dhellemmes S, Wissinger B, et al. (2009) 
TRPM1 is mutated in patients with autosomal-recessive complete congenital stationary night blindness. Am J Hum Genet 85:720-729.

Bech-Hansen NT, Naylor MJ, Maybaum TA, Sparkes RL, Koop B, Birch DG, Bergen AA, Prinsen CF, Polomeno RC, Gal A, Drack AV, Musarella MA, Jacobson SG, Young RS, Weleber RG (2000) Mutations in NYX, encoding the leucine-rich proteoglycan nyctalopin, cause X-linked complete congenital stationary night blindness. Nat Genet 26:319-323.

Beqollari D, Betzenhauser MJ, Kammermeier PJ (2009) Altered G-protein coupling in an mGluR6 point mutant associated with congenital stationary night blindness. Mol Pharmacol 76:992-997.

Berger W, Kloeckener-Gruissem B, Neidhardt J (2010) The molecular basis of human retinal and vitreoretinal diseases. Prog Retin Eye Res 29:335-375.

Bürckstümmer T, Bennett KL, Preradovic A, Schütze G, Hantschel O, Superti-Furga G, Bauch A (2006) An efficient tandem affinity purification procedure for interaction proteomics in mammalian cells. Nat Methods 3:1013-1019.

Cao Y, Song H, Okawa H, Sampath AP, Sokolov M, Martemyanov KA (2008) Targeting of RGS7/Gbeta5 to the dendritic tips of ON-bipolar cells is independent of its association with membrane anchor R7BP. J Neurosci 28:10443-10449.

Cao Y, Masuho I, Okawa H, Xie K, Asami J, Kammermeier PJ, Maddox DM, Furukawa T, Inoue T, Sampath AP, Martemyanov KA (2009) Retinaspecific GTPase accelerator RGS11/G beta 5S/R9AP is a constitutive heterotrimer selectively targeted to mGluR6 in ON-bipolar neurons. J Neurosci 29:9301-9313.

Dhingra A, Lyubarsky A, Jiang M, Pugh EN Jr, Birnbaumer L, Sterling P, Vardi N (2000) The light response of ON bipolar neurons requires Gao. J Neurosci 20:9053-9058.

Fain GL, Hardie R, Laughlin SB (2010) Phototransduction and the evolution of photoreceptors. Curr Biol 20:R114-R124.

Field GD, Rieke F (2002) Nonlinear signal transfer from mouse rods to bipolar cells and implications for visual sensitivity. Neuron 34:773-785.

Gregg RG, Mukhopadhyay S, Candille SI, Ball SL, Pardue MT, McCall MA, Peachey NS (2003) Identification of the gene and the mutation responsible for the mouse nob phenotype. Invest Ophthalmol Vis Sci 44:378-384.

Gregg RG, Kamermans M, Klooster J, Lukasiewicz PD, Peachey NS, Vessey KA, McCall MA (2007) Nyctalopin expression in retinal bipolar cells restores visual function in a mouse model of complete X-linked congenital stationary night blindness. J Neurophysiol 98:3023-3033.

Hsu SY, Kudo M, Chen T, Nakabayashi K, Bhalla A, van der Spek PJ, van Duin M, Hsueh AJ (2000) The three subfamilies of leucine-rich repeat-containing G protein-coupled receptors (LGR): identification of LGR6 and LGR7 and the signaling mechanism for LGR7. Mol Endocrinol 14:1257-1271.

Ko J, Kim E (2007) Leucine-rich repeat proteins of synapses. J Neurosci Res $85: 2824-2832$.

Koike C, Obara T, Uriu Y, Numata T, Sanuki R, Miyata K, Koyasu T, Ueno S, Funabiki K, Tani A, Ueda H, Kondo M, Mori Y, Tachibana M, Furukawa T (2010) TRPM1 is a component of the retinal ON bipolar cell transduction channel in the mGluR6 cascade. Proc Natl Acad Sci U S A 107:332-337.

Lambert S, Drews A, Rizun O, Wagner TF, Lis A, Mannebach S, Plant S, Portz M, Meissner M, Philipp SE, Oberwinkler J (2011) Transient receptor potential melastatin 1 (TRPM1) is an ion-conducting plasma membrane channel inhibited by zinc ions. J Biol Chem 286:12221-12233.

Leroy BP, Budde BS, Wittmer M, De Baere E, Berger W, Zeitz C (2009) A common NYX mutation in Flemish patients with X linked CSNB. Br J Ophthalmol 93:692-696.

Maddox DM, Vessey KA, Yarbrough GL, Invergo BM, Cantrell DR, Inayat S, Balannik V, Hicks WL, Hawes NL, Byers S, Smith RS, Hurd R, Howell D, Gregg RG, Chang B, Naggert JK, Troy JB, Pinto LH, Nishina PM, McCall MA (2008) Allelic variance between GRM6 mutants, Grm6nob3 and Grm6nob4 results in differences in retinal ganglion cell visual responses. J Physiol 586:4409-4424.

Martemyanov KA, Yoo PJ, Skiba NP, Arshavsky VY (2005) R7BP, a novel neuronal protein interacting with RGS proteins of the R7 family. J Biol Chem 280:5133-5136.

Masu M, Iwakabe H, Tagawa Y, Miyoshi T, Yamashita M, Fukuda Y, Sasaki H, Hiroi K, Nakamura Y, Shigemoto R (1995) Specific deficit of the ON response in visual transmission by targeted disruption of the mGluR6 gene. Cell 80:757-765.

Masuho I, Celver J, Kovoor A, Martemyanov KA (2010) Membrane anchor
R9AP potentiates GTPase-accelerating protein activity of RGS11/Gbeta5 complex and accelerates inactivation of the mGluR6-Go signaling. J Biol Chem 285:4781-4787.

Morgans CW, Ren G, Akileswaran L (2006) Localization of nyctalopin in the mammalian retina. Eur J Neurosci 23:1163-1171.

Morgans CW, Zhang J, Jeffrey BG, Nelson SM, Burke NS, Duvoisin RM, Brown RL (2009) TRPM1 is required for the depolarizing light response in retinal ON-bipolar cells. Proc Natl Acad Sci U S A 106:19174-19178.

Morgans CW, Brown RL, Duvoisin RM (2010) TRPM1: the endpoint of the mGluR6 signal transduction cascade in retinal ON-bipolar cells. Bioessays 32:609-614.

Nakajima Y, Iwakabe H, Akazawa C, Nawa H, Shigemoto R, Mizuno N, NakanishiS (1993) Molecular characterization of a novel retinal metabotropic glutamate receptor mGluR6 with a high agonist selectivity for L-2-amino-4phosphonobutyrate. J Biol Chem 268:11868-11873.

Nakamura M, Sanuki R, Yasuma TR, Onishi A, Nishiguchi KM, Koike C, Kadowaki M, Kondo M, Miyake Y, Furukawa T (2010) TRPM1 mutations are associated with the complete form of congenital stationary night blindness. Mol Vis 16:425-437.

Nawy S (1999) The metabotropic receptor mGluR6 may signal through $\mathrm{G}(\mathrm{o})$, but not phosphodiesterase, in retinal bipolar cells. J Neurosci 19:2938-2944.

Oancea E, Vriens J, Brauchi S, Jun J, Splawski I, Clapham DE (2009) TRPM1 forms ion channels associated with melanin content in melanocytes. Sci Signal 2:ra21.

O'Connor E, Allen LE, Bradshaw K, Boylan J, Moore AT, Trump D (2006) Congenital stationary night blindness associated with mutations in GRM6 encoding glutamate receptor mGluR6. Br J Ophthalmol 90:653-654.

Okawa H, Sampath AP (2007) Optimization of single-photon response transmission at the rod-to-rod bipolar synapse. Physiology (Bethesda) 22:279-286.

Poopalasundaram S, Erskine L, Cheetham ME, Hardcastle AJ (2005) Focus on molecules: nyctalopin. Exp Eye Res 81:627-628.

Posokhova E, Song H, Belcastro M, Higgins L, Bigley LR, Michaud NA, Martemyanov KA, Sokolov M (2011) Disruption of the Chaperonin containing TCP-1 function affects protein networks essential for rod outer segment morphogenesis and survival. Mol Cell Proteomics 10:M110.000570.

Pugh EN Jr (2006) RGS expression level precisely regulates the duration of rod photoresponses. Neuron 51:391-393.

Sharpe LT, Stockman A (1999) Rod pathways: the importance of seeing nothing. Trends Neurosci 22:497-504.

Shen Y, Heimel JA, Kamermans M, Peachey NS, Gregg RG, Nawy S (2009) A transient receptor potential-like channel mediates synaptic transmission in rod bipolar cells. J Neurosci 29:6088-6093.

Shevchenko A, Tomas H, Havlis J, Olsen JV, Mann M (2006) In-gel digestion for mass spectrometric characterization of proteins and proteomes. Nat Protoc 1:2856-2860.

Snellman J, Kaur T, Shen Y, Nawy S (2008) Regulation of ON bipolar cell activity. Prog Retin Eye Res 27:450-463.

Taylor WR, Smith RG (2004) Transmission of scotopic signals from the rod to rod-bipolar cell in the mammalian retina. Vision Res 44:3269-3276.

Tsunoda S, Zuker CS (1999) The organization of INAD-signaling complexes by a multivalent PDZ domain protein in Drosophila photoreceptor cells ensures sensitivity and speed of signaling. Cell Calcium 26:165-171.

van Genderen MM, Bijveld MM, Claassen YB, Florijn RJ, Pearring JN, Meire FM, McCall MA, Riemslag FC, Gregg RG, Bergen AA, Kamermans M (2009) Mutations in TRPM1 are a common cause of complete congenital stationary night blindness. Am J Hum Genet 85:730-736.

Vardi N, Dhingra A, Zhang L, Lyubarsky A, Wang TL, Morigiwa K (2002) Neurochemical organization of the first visual synapse. Keio J Med 51:154-164.

Wang T, Montell C (2007) Phototransduction and retinal degeneration in Drosophila. Pflugers Arch 454:821-847.

Yan J, Aldrich RW (2010) LRRC26 auxiliary protein allows BK channel activation at resting voltage without calcium. Nature 466:513-516.

Zeitz C, van Genderen M, Neidhardt J, Luhmann UF, Hoeben F, Forster U, Wycisk K, Mátyás G, Hoyng CB, Riemslag F, Meire F, Cremers FP, Berger W (2005) Mutations in GRM6 cause autosomal recessive congenital stationary night blindness with a distinctive scotopic $15 \mathrm{~Hz}$ flicker electroretinogram. Invest Ophthalmol Vis Sci 46:4328-4335. 